\title{
Postherpetic abdominal pseudohernia
}

\author{
Volume 7 Issue 7 - 2017
}

\section{Clinical image}

With great interest we would like to share and discuss another differential diagnosis of postherpetic neuropathy in a middle-aged adult: Postherpetic abdominal pseudohernia. The present case refers to an A 53-year-old man presented with a 4-day installation of left abdominal wall protrusion intensified with Valsalva maneuver associated with skin painful dried eruptions. Two weeks before, he underwent treatment for Herpes Zoster in the left flank. Abdominal sonography and MRI of thoracic, lumbar spine and abdomen were normal. Electromyography showed denervation in bilateral (T10-11) paraspinal muscles. After 6months, he had a complete resolution of the clinical course (Figure). ${ }^{1}$ Postherpetic abdominal pseudohernia may be interpreted as an uncommon viral involvement of ventral roots and associated with good prognosis. ${ }^{2}$ Attention to this condition may help neurologists make correct diagnoses.
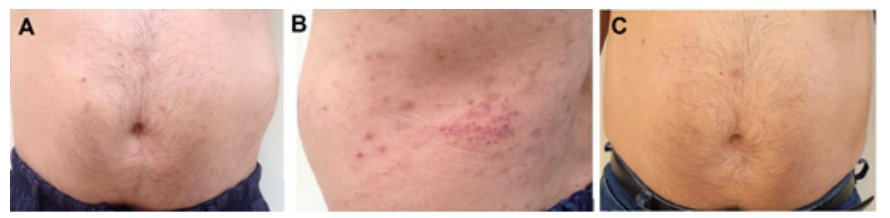

Figure I Postherpetic abdominal pseudohernia

A) Protrusion of the left abdominal wall, B) Skin dried eruptions in the left flank, C) Complete resolution of the pseudohernia.

\section{Author contributions}

These authors contributed equally to the manuscript.

a. Karine Takizawa- Acquisition of data.

b. José Roberto Paes de Almeida- Study supervision.

c. Joseph Bruno Bidin Brooks- Study concept and design.

d. Daniela Cechi- Analysis and interpretation.
Karine Takizawa,' José Roberto Paes de Almeida,, 2 Joseph Bruno Bidin Brooks, ${ }^{3}$ Daniela Cechi,' Darlene Silva Polito'

'Department of Dermatology, UNILUS- Centro Universitário Lusíada, Brazil

${ }^{2}$ Department of Dermatology, UNIMES- Universidade

Metropolitana de Santos, Brazil

${ }^{3}$ Department of Neurology, Department of Function and Structure, UNIMES- Universidade Metropolitana de Santos, Brazil

Correspondence: Joseph Bruno Bidin Brooks, Irmandade Santa Casa de Misericórida de Santos, Avenidade Dr Claudio Luiz da Costa 5, Santos, São Paulo, Brazil, I I75-9, Tel +55-1332020600,Email joseph3b@gmail.com

Received:September 12, 2017 | Published: December 19, 2017

e. Darlene Silva Polito- Critical revision of the manuscript for important intellectual content.

\section{Conflict of interest}

None.

\section{Acknowledgements}

None.

\section{Funding}

None.

\section{References}

1. Tagg NT, Tsao JW. Images in clinical medicine. Abdominal pseudohernia due to herpes zoster. $N$ Engl J Med. 2006;355(1):e1.

2. Hung CT, Wang WM. Postherpetic pseudohernia. CMAJ. 2012;184(3):E202. 